\title{
Structure of chlorine isotopes populated by heavy ion transfer reactions
}

\author{
S. Szilner, ${ }^{1}$ L. Corradi, ${ }^{2}$ F. Haas, ${ }^{3}$ G. Pollarolo,,${ }^{4}$ L. Angus, ${ }^{5}$ S. Beghini, ${ }^{6}$ M. Bouhelal, ${ }^{7}$ R. Chapman, ${ }^{5}$ E. Caurier, ${ }^{3}$ S. Courtin, ${ }^{3}$ \\ E. Farnea, ${ }^{6}$ E. Fioretto, ${ }^{2}$ A. Gadea, ${ }^{2,8}$ A. Goasduff, ${ }^{3}$ D. Jelavić-Malenica, ${ }^{1}$ V. Kumar, ${ }^{5}$ S. Lunardi, ${ }^{6}$ N. Mărginean, ${ }^{2,9}$ \\ D. Mengoni, ${ }^{6}$ T. Mijatović, ${ }^{1}$ G. Montagnoli, ${ }^{6}$ F. Recchia, ${ }^{6}$ E. Sahin, ${ }^{2}$ M.-D. Salsac, ${ }^{3,10}$ F. Scarlassara ${ }^{6}$ J. F. Smith, ${ }^{5}$ N. Soić, ${ }^{1}$ \\ A. M. Stefanini, ${ }^{2}$ C. A. Ur, ${ }^{6}$ and J. J. Valiente-Dobón ${ }^{2}$ \\ ${ }^{1}$ Ruđer Bošković Institute, Zagreb, Croatia \\ ${ }^{2}$ INFN - Laboratori Nazionali di Legnaro, Legnaro, Italy \\ ${ }^{3} I P H C, C N R S / I N 2 P 3$ and Université de Strasbourg, Strasbourg, France \\ ${ }^{4}$ INFN and Università di Torino, Italy \\ ${ }^{5}$ School of Engineering, University of the West of Scotland, Paisley, United Kingdom \\ ${ }^{6}$ INFN and Università di Padova, Padova, Italy \\ ${ }^{7}$ Laboratoire de Physique Appliquée et Théorique, Université de Tébessa, Tébessa, Algérie \\ ${ }^{8}$ IFIC, CSIC-Universidad de Valencia, Valencia, Spain \\ ${ }^{9}$ Horia Hulubei National Institute of Physics and Nuclear Engineering, Bucharest, Romania \\ ${ }^{10}$ CEA, Centre de Saclay, IRFU/SPhN, Gif-sur-Yvette, France
}

(Received 27 November 2012; revised manuscript received 23 April 2013; published 20 May 2013)

\begin{abstract}
Neutron-rich chlorine isotopes were populated in the ${ }^{40} \mathrm{Ar}+{ }^{208} \mathrm{~Pb}$ multiple transfer reaction via the $-1 p$ channels in a fragment- $\gamma$ coincident measurement employing the Prisma-Clara setup. New $\gamma$ transitions have been identified and, together with already available data, level schemes have been revised and compared with the results of large-scale $s d$ - $p f$ shell-model calculations. The evolution of the energy splitting between the $s_{1 / 2}$ and $d_{3 / 2}$ orbitals and the increased mixing of different proton configurations in the populated $\mathrm{Cl}$ isotopic chain have been deduced by inspecting the shell-model wave functions.
\end{abstract}

DOI: 10.1103/PhysRevC.87.054322

PACS number(s): 25.70.Hi, 23.20.Lv, 21.60.Cs, 29.30.Aj

\section{INTRODUCTION}

In the nuclear region ranging from $\mathrm{Si}$ to $\mathrm{Ca}$ and with neutron number between $N=20$ and $N=28$, a rapid change of nuclear structure has been observed [1-14]. The observed collectivity, shape coexistence, and configuration mixing were discussed in terms of the erosion of the $N=28$ shell gap and of the $Z=16$ subshell closure [15-21]. For example, in the vicinity of the neutron-rich $\mathrm{S}$ isotopes, a high degree of collectivity was reported, as first inferred from the behavior of the proton $s_{1 / 2}$ and $d_{3 / 2}$ orbitals in the $\mathrm{K}$ isotopic chain [10]. The evolution of the energy splitting among the two orbitals as a function of the neutron number enlightened the microscopic effects affecting the changes in the single-particle levels and relative contributions of the central and tensor components of the nucleon-nucleon interactions [22]. The effect of the near degeneracy of the $s_{1 / 2}$ and $d_{3 / 2}$ orbitals and of the induced proton correlations may be probed in the $\mathrm{Cl}(Z=17)$ isotopes $[8,10,23]$. These may be considered as having three proton holes with respect to the closed $Z=20 s d$ shell.

$\mathrm{Cl}$ ions have been produced in the ${ }^{40} \mathrm{Ar}+{ }^{208} \mathrm{~Pb}$ reaction via the one proton stripping transfer channels, and new $\gamma$ transitions have been assigned to the isotopes with neutron number ranging from $N=20\left({ }^{37} \mathrm{Cl}\right)$ to $N=26\left({ }^{43} \mathrm{Cl}\right)$. We benefited from the use of multinucleon transfer reactions, which are known to be a powerful tool for the study of nuclear structure properties of nuclei moderately far from stability (see Refs. [24,25] and references therein). In this way it was possible to investigate chains of isotopes populated, at once, by the same reaction mechanism. Thus, poorly known (neutron-rich) isotopes could be more easily compared with better known ones, making the attribution of level properties more reliable when coincidences between particles and single $\gamma$ are available. This was shown in different cases [26-29], where electromagnetic transitions coming from the decay of specific nuclei have been identified by exploiting the mass and nuclear charge resolution of binary products with large solid angle magnetic spectrometers coupled to large $\gamma$ arrays. In particular, the presently used reaction has been recently employed to study shell properties in Ar isotopic chain populated via neutron pickup channels, where the newly observed states have been used to consistently follow the evolution of collectivity in odd Ar isotopes [28].

\section{EXPERIMENT AND DATA ANALYSIS}

An ${ }^{40} \mathrm{Ar}$ beam has been extracted by an ECR ion source and accelerated by means of the superconducting ALPI-Linac accelerator of the Laboratory Nazionali di Legnaro at $E_{\mathrm{lab}}=$ $255 \mathrm{MeV}$ onto a $300 \mu \mathrm{g} / \mathrm{cm}^{2}{ }^{208} \mathrm{~Pb}$ target. Projectile-like fragments have been identified with the large solid angle magnetic spectrometer Prisma [30,31] placed in the vicinity of the grazing angle $\left(\theta_{\mathrm{lab}}=54^{\circ}\right)$. We here briefly recall the Prisma detector system. A position-sensitive microchannel plate detector [32] was placed at the entrance of the spectrometer, providing a start signal for time-of-flight measurements and position signals. Ions pass through the optical elements of the spectrometer (a quadrupole and a dipole) and enter a focal plane [33] whose first part is made of a parallel plate of multiwire type, providing timing and position signals with resolutions similar to the entrance detector. At the end of 


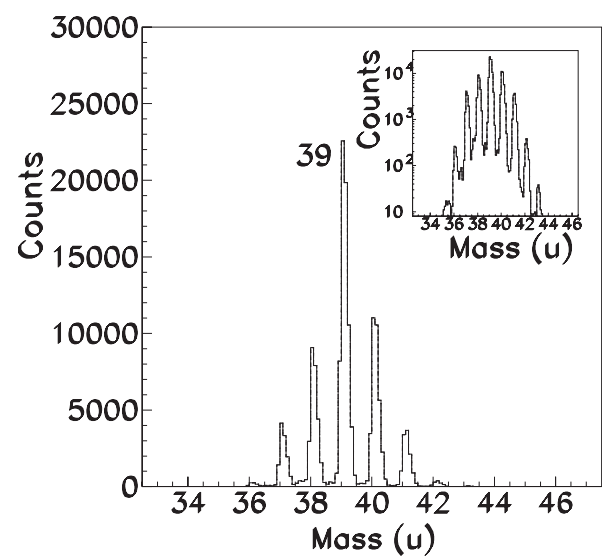

FIG. 1. Mass distribution of chlorine isotopes populated in ${ }^{40} \mathrm{Ar}+{ }^{208} \mathrm{~Pb}$ at $E_{\mathrm{lab}}=255 \mathrm{MeV}$ and at $\theta_{\mathrm{lab}}=54^{\circ}$ in coincidence with $\gamma$ rays. A mass resolution of $\Delta A / A \sim 1 / 160$ has been obtained. The inset displays the same mass distribution in logarithmic scale.

the focal plane an array of transverse field multiparametric ionization chamber was located, providing nuclear charge (via $\Delta E$ ) and total energy $(E)$. The described detector system gives all the necessary information for the complete ion identification, which was performed via an event-by-event reconstruction of the trajectory inside the magnetic elements [31].

To illustrate the achieved resolution, the mass distribution of chlorine isotopes populated by one-proton stripping reactions

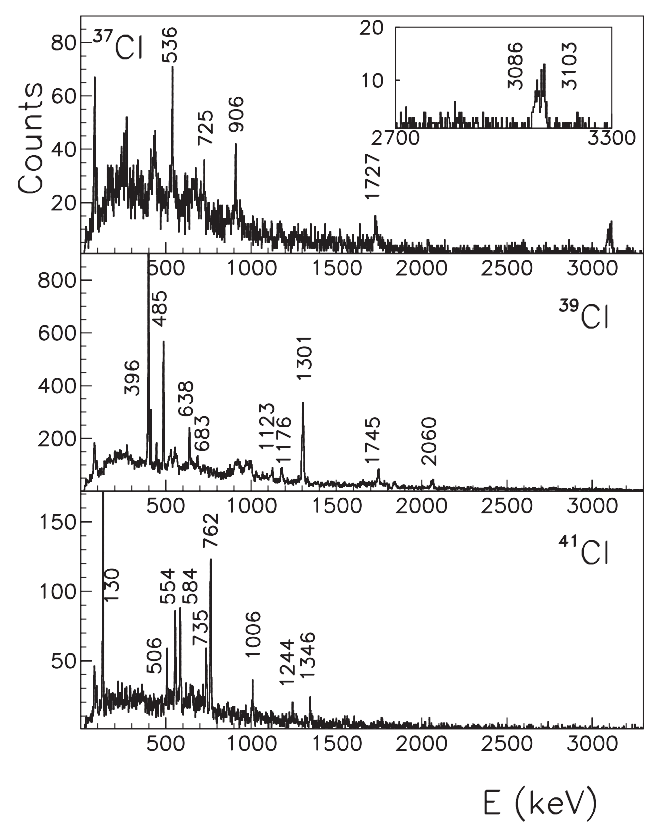

FIG. 2. Doppler corrected $\gamma$-ray spectra for ${ }^{37,39,41} \mathrm{Cl}$ populated in ${ }^{40} \mathrm{Ar}+{ }^{208} \mathrm{~Pb}$ by detecting the $-1 p-2 n,-1 p$, and $-1 p+1 n$ channels in Prisma and the coincident $\gamma$ rays in Clara. The strongest transitions are labeled with $E_{\gamma}$; see Ref. [38] and Table I. Strong $\gamma$ transitions, not labeled in the lower energy part of the spectra, belong to the wrongly Doppler corrected $\gamma$ rays of the heavy partners. The inset displays the enlarged energy region in vicinity of $\sim 3100 \mathrm{keV}$ of ${ }^{37} \mathrm{Cl}$. has been plotted in Fig. 1. It is a well known rule that dominantly proton stripping and neutron pickup occur in transfer processes involving stable projectiles [34,35]. As a consequence, projectile nuclei tend to gain some neutrons while loosing protons, populating the region to the "right" of the stability valley. The origin of this mechanism lies in characteristics of reaction $Q$ values [25]. As one takes heavier isotopes of a given projectile a transition toward the opposite situation should eventually take place [36]. In the actual ${ }^{40} \mathrm{Ar}+{ }^{208} \mathrm{~Pb}$ reaction, by exploiting the most neutron-rich stable argon isotope one populates nuclei toward the neutron-rich side, not too far from the projectile. In the case of the chlorine isotopes the mass distribution is rather symmetric around ${ }^{39} \mathrm{Cl}$ (the pure $-1 p$ channel).

The $\gamma$ rays were detected with the Clara array [37], which consisted of 24 high-purity Ge clover-type detectors situated in the hemisphere opposite to Prisma. The total photopeak efficiency was $\sim 3 \%$ for $E=1.33 \mathrm{MeV}$. The fragment- $\gamma$ coincidence obtained from the coupling of Clara with Prisma allows us to attribute to each specific reaction product its characteristic $\gamma$ rays. Doppler $\gamma$ correction is obtained from the knowledge of the reconstructed fragment velocity vector, with typical $\gamma$-ray energy resolutions of $0.6 \%$ to $0.9 \%$ over the whole velocity distribution.

The $\gamma$-ray spectra of the odd-even $\mathrm{Cl}$ isotopes, i.e., ${ }^{37,39,41} \mathrm{Cl}$ corresponding to the $-1 p-2 n,-1 p$, and $-1 p+2 n$ channels, respectively, are shown in Fig. 2 whereas those of the odd-odd $\mathrm{Cl}$ isotopes, i.e., ${ }^{38,40,42} \mathrm{Cl}$ corresponding to the $-1 p-1 n,-1 p+1 n$, and $-1 p+3 n$ channels, respectively, are shown in Fig. 3. The used method allowed the assignment,

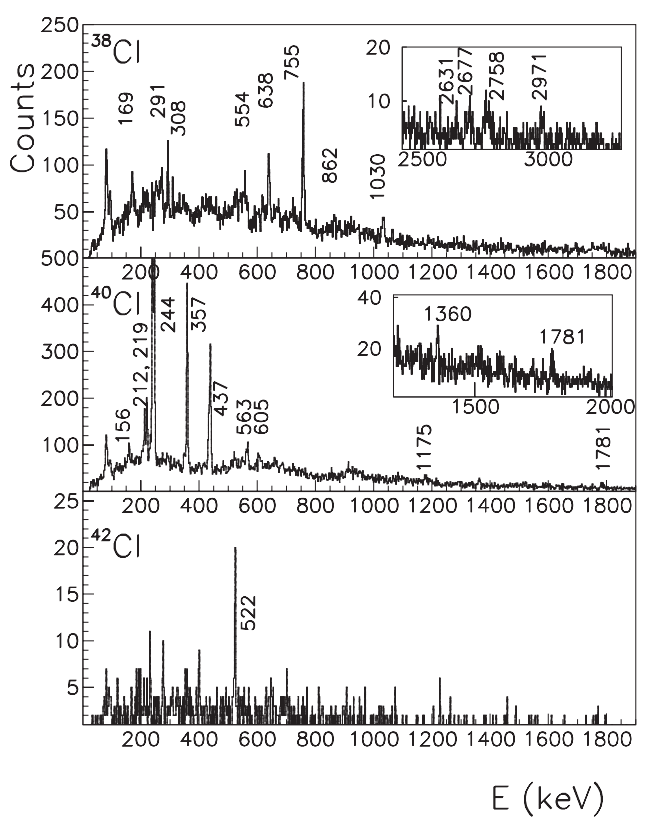

FIG. 3. Doppler corrected $\gamma$-ray spectra for ${ }^{38,40,42} \mathrm{Cl}$ populated in ${ }^{40} \mathrm{Ar}+{ }^{208} \mathrm{~Pb}$ by detecting the $-1 p-1 n,-1 p+1 n$, and $-1 p+3 n$ channels in Prisma and the coincident $\gamma$ rays in Clara. The strongest transitions are labeled with $E_{\gamma}$; see Ref. [38] and Table II. Strong $\gamma$ transitions, not labeled in the lower energy part of the spectra, belong to the wrongly Doppler corrected $\gamma$ rays of the heavy partners. The insets display the higher energy regions in ${ }^{38} \mathrm{Cl}$ and ${ }^{40} \mathrm{Cl}$. 
in some cases for the first time, of $\gamma$ rays to a specific nucleus. The statistics of the $\gamma-\gamma$ data of Clara were generally insufficient to prove coincidence relationships among the $\gamma$ rays assigned to each isotope. The level schemes of the chlorine isotopes, presented and discussed in the following sections, have been updated based mainly on systematics with neighboring nuclei, and when available, on $\gamma-\gamma$ coincidence data from literature. Large-scale shell-model calculations have been helpful in suggestions of spin and parities of the newly proposed states.

\section{LEVEL SCHEMES OF CI ISOTOPES AND SHELL-MODEL CALCULATIONS}

For $\mathrm{Cl}$ isotopes lying close to the stability line, substantial experimental information exists [38] from $(d, p)$ and deep inelastic reactions performed with the "thick-target" technique. However, going further away to more neutron-rich $\mathrm{Cl}$ nuclei, the information is much more scarce, mainly coming from $\beta$-decay studies in nuclei produced via fragmentation reactions or from ${ }^{36} \mathrm{~S}+{ }^{208} \mathrm{~Pb}$ multinucleon transfer reactions. In the present work, the $\mathrm{Cl}$ isotopes are produced via a multinucleon transfer reaction starting from the most neutron-rich stable ${ }^{40} \mathrm{Ar}$ isotope, populating a more favorable proton stripping region, in terms of yield and $Q$ value.

In this section we present the level schemes of chlorine isotopes, populated in our study, divided into odd-even and odd-odd isotopes, and the shell-model (SM) calculations performed to interpret the data. The SM calculations have been performed using the most recent $s d-p f$ residual interaction (SDPF-U) [39]. The valence space for the protons includes the full $s d$ shell, covering from $Z=8$ to $Z=20$, while for the neutrons it includes the full $s d$ and $p f$ shells, from $N=8$ to $N=40$. We remind that below $N=20$ these calculations reduce to the pure $s d$ shell. Thus, the SM calculations are unrestricted in the full $s d$ shell for the protons and the full $p f$ shell for the neutrons. The effective interaction is composed of three blocks: $s d-s d, s d-p f$ (proton-neutron), and $p f-p f$ (neutron-neutron). The states, described in the $0 \hbar \omega$ space [40], are compared with the experimental findings for all chlorine isotopes with $N>20$. For the ${ }^{37} \mathrm{Cl}(N=20)$ case we used the recently developed $(0+1) \hbar \omega$ interaction called PSDPF [41]. This interaction uses the full $p$-sd-pf space, allowing one nucleon jump from $p$ to $s d$ or from $s d$ to $p f$ shells, in order to describe one-particle-one-hole intruder states throughout the $s d$ shell.

Special emphasis has been given on the dominant components of the wave function of the strongly populated states and how they evolve towards the more neutron-rich isotopes. The importance of the $d_{3 / 2}$ and $s_{1 / 2}$ proton orbitals and of the promotion of the neutrons above the $N=28$ shell gap in the development of the collectivity will be then extensively discussed in Sec. IV.

\section{A. Structure of odd-even $\mathrm{Cl}$ isotopes}

The transitions observed in odd-even $\mathrm{Cl}$ isotopes, which include also the adopted spin and parity [38], are depicted in the level schemes of Figs. 4, 5, and 7 together with the shell-model (SM) calculation [42]

The ${ }^{37} \mathrm{Cl}$ nucleus was recently studied using several heavy ion induced reactions [43-47], where new $\gamma$ transitions were reported. In particular, this isotope was populated in ${ }^{160} \mathrm{Gd}+{ }^{36} \mathrm{~S}$ and ${ }^{160} \mathrm{Gd}+{ }^{37} \mathrm{Cl}$ deep-inelastic collisions in thick-target measurements where $\gamma-\gamma$ coincidences were obtained [43]. The transitions $E_{\gamma}=536,906$, and $3103 \mathrm{keV}$ were attributed to ${ }^{37} \mathrm{Cl}$, and described as a cascade $11 / 2^{-} \rightarrow$ $9 / 2^{-} \rightarrow 7 / 2^{-} \rightarrow 3 / 2_{\text {g.s. }}^{+}$(although, the evidence of the spinparity assignments was not discussed). The same $\gamma$ transitions, uniquely attributed to ${ }^{37} \mathrm{Cl}$, have been observed in our work through fragment- $\gamma$ coincidences. ${ }^{37} \mathrm{Cl}$ was also populated via the $+1 p$ channel in the ${ }^{36} \mathrm{~S}+{ }^{208} \mathrm{~Pb}$ transfer reaction performed in a fragment $-\gamma$ coincident measurement with Prisma + Clara [45]. The results of that work agree with the level scheme of Fig. 4. By using the fusion evaporation reaction ${ }^{24} \mathrm{Mg}\left({ }^{16} \mathrm{O}, 3 p\right)$ [44], the level scheme of ${ }^{37} \mathrm{Cl}$ was extended up to an excitation energy of $\sim 17 \mathrm{MeV}$ with spins and parities up to $\left(29 / 2^{+}\right)$and $\left(27 / 2^{-}\right)$.

The levels of ${ }^{37} \mathrm{Cl}$ are compared with $(0+1) \hbar \omega$ calculations with the PSDPF interaction for both positive (natural) and negative (intruder) parity states in Fig. 4. In general, the results of SM calculations are in excellent agreement with the experimental level scheme.

Concerning the experimental findings for ${ }^{37} \mathrm{Cl}$, one observes an almost symmetrical distribution of the population strength of the positive and negative parity bands. The decay of the $1 / 2^{+}$and $5 / 2^{+}$states dominates the positive parity band. Inferring the SM wave functions, we explored in more detail the dominant component of the three lowest positive parity states. For the $3 / 2_{\text {g.s. }}^{+}$and $1 / 2^{+}$states, about $93 \%$ of squared amplitudes of their wave functions are with a proton in $d_{3 / 2}$ and $s_{1 / 2}$, respectively. These configurations have a filled neutron shell, providing a semimagic nature of the ${ }^{37} \mathrm{Cl}$ nucleus evident in the level scheme with a large gap between the ground and first excited states. In the first $5 / 2^{+}$state, $93 \%$ is in the $\pi\left(d_{5 / 2}\right)^{6}\left(s_{1 / 2}\right)^{1}\left(d_{3 / 2}\right)^{2}$, and only about $5 \%$ in the $\pi\left(d_{5 / 2}\right)^{5}\left(s_{1 / 2}\right)^{1}\left(d_{3 / 2}\right)^{3}$ proton configurations. In general, the wave functions of the strongly populated states are rather pure, and their main component contributes to the squared amplitudes of wave functions with more than $90 \%$. Thus, transitions between positive and negative parity states turn out to be rather weak.

The negative parity band is the result of the promotion of one nucleon from the $s d$ to the $f p$ shell. Here, for the ${ }^{37} \mathrm{Cl}$ nucleus, this band consists of the series of states with spin-parity $13 / 2^{-}, 11 / 2^{-}, 9 / 2^{-}$, and $7 / 2^{-}$. Indications of transitions corresponding to the direct decays of $5 / 2^{-}$and $9 / 2^{-}$to the ground state are weakly observed in the $\gamma$ spectra and are, thus, represented by dashed lines in Fig. 4. Their weak intensity is consistent with the known branching ratios and expected Clara efficiency for high energy $\gamma$ rays [37] $\left(E_{\gamma}=3.7\right.$ and $\left.4.0 \mathrm{MeV}\right)$. For the $13 / 2^{-}, 11 / 2^{-}$, and $9 / 2^{-}$ states, the dominant component of the wave function is the expected proton configuration $\left(s_{1 / 2}\right)^{2}\left(d_{3 / 2}\right)^{1}$ coupled to a neutron promoted to the $f_{7 / 2}$ orbital $(68 \%, 60 \%$, and $42 \%$, for $13 / 2^{-}, 11 / 2^{-}$, and $9 / 2^{-}$, respectively). The other important component concerns the proton rearrangement within $d_{3 / 2}$ and 

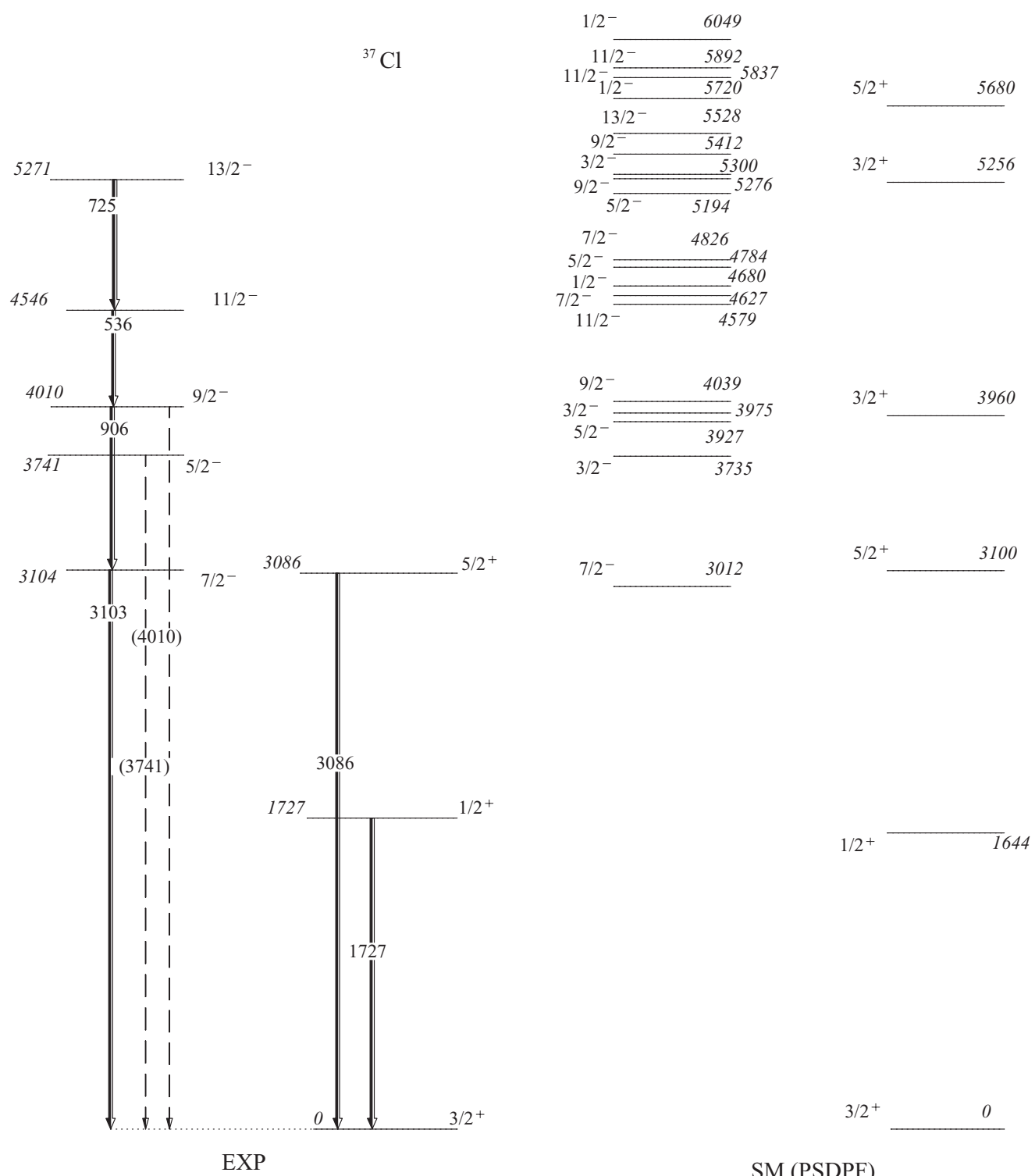

SM (PSDPF)

FIG. 4. The experimental negative and positive parity (left) and SM calculated (PSDPF interaction) negative and positive parity (right) level schemes of ${ }^{37} \mathrm{Cl}$, populated via the $-1 p-2 n$ channel. Relative $\gamma$-ray intensities (left) are indicated by the widths of the arrows (see text for dashed lines). Only the states populated in our measurement are plotted. The energy, spin and parity of levels, their branching ratios, and energy of transitions, where known, are as in Ref. [38]. SM calculations (using PSDPF interaction) with energies up to $\sim 5.6 \mathrm{MeV}$ for the positive and up to $\sim 6 \mathrm{MeV}$ for the negative parities are plotted (right).

$s_{1 / 2}$, for the $13 / 2^{-}$and $11 / 2^{-}$states. For the $9 / 2^{-}$state, the component with closed neutron shell and the promotion of a proton to the $f p$ shell becomes more important, and even the largest for the $7 / 2^{-}$state $(45 \%)$. The relevance of this component, also observed in neighboring nuclei, was extensively discussed in Ref. [1] and references therein.

The $\gamma$ spectrum in coincidence with ${ }^{39} \mathrm{Cl}$ is shown in the middle panel of Fig. 2. The strongest $\gamma$ lines correspond to the decays of the $1 / 2^{+}$and $5 / 2^{+}$states, similarly to ${ }^{37} \mathrm{Cl}$. We have observed the $\gamma$ decays of all known states up to $\left(9 / 2^{+}\right)$at $2423.7 \mathrm{keV}$, with populated strengths depending on the transfer mechanism via excitation energy, spin, and structure of corresponding states. The population of the yrast states in the positive parity band is followed up to $\left(15 / 2^{+}\right)$ at $3517.2 \mathrm{keV}$. Besides the yrast states, we also observed the decay of the second and third $5 / 2^{+}$states, and the second $1 / 2^{+}$ state. Studies of higher spin states up to $15 / 2^{+}$were reported in Ref. [43], while a very extensive study of ${ }^{39} \mathrm{Cl}$ was also carried out in $\beta$-decay and light ion induced transfer reactions [48,49].

The level scheme of ${ }^{39} \mathrm{Cl}$, shown in Fig. 5, besides our data, is based on the information available from the Paisley group measurements [45-47]. In particular, the ${ }^{37} \mathrm{Cl}+{ }^{160} \mathrm{Gd}$ reaction [47] has been used to establish the level scheme of ${ }^{39} \mathrm{Cl}$, although the spins and parities were only partially assigned. $\gamma-\gamma$ coincidence data, measured with the GASP $\gamma$ array [46] in the ${ }^{36} \mathrm{~S}+{ }^{176} \mathrm{Yb}$ reaction, 


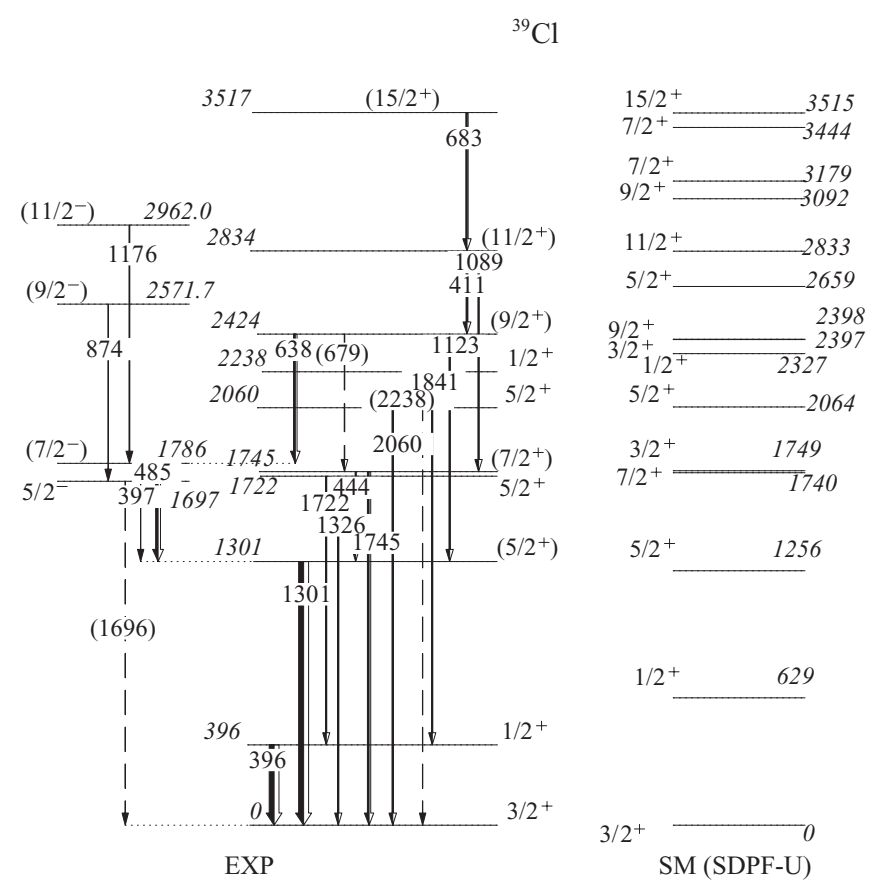

FIG. 5. Experimental negative and positive parity (left) and SM calculated (right) level scheme of ${ }^{39} \mathrm{Cl}$, populated via the $-1 p$ channel. SM calculations have been done by using the SDPF-U interaction in the $0 \hbar \omega$ space, therefore only the positive parity states are described and they are plotted with energies up to $\sim 3600 \mathrm{keV}$ (right). Relative $\gamma$-ray intensities (left) are indicated by the widths of the arrows. Only the states populated in our measurement are plotted (solid lines). The energy, spin and parity of levels, their branching ratios (with tabulated $\gamma$ intensity $I_{\gamma} \geqslant 10$ ), and energy of transitions, where known, are as in Ref. [38]. Some of the weaker intensity $\gamma$ rays were not observed in our measurement with sufficient statistical accuracy, thus they are plotted as dashed lines. The state at $2572 \mathrm{keV}$ is tabulated as $(5 / 2,7 / 2,9 / 2)^{-}$, where we have preference for $J^{\pi}=9 / 2^{-}$, the $9 / 2^{-}$being a yrast state. The $\gamma$ ray with $E_{\gamma}=1176.4 \mathrm{keV}$ has been attributed to the $\left(11 / 2^{-}\right) \rightarrow\left(7 / 2^{-}\right)$ transition.

have been, as well, used to build the level scheme plotted in Fig. 5.

In the fragment- $\gamma$ Prisma-Clara coincidence measurement [45], ${ }^{39} \mathrm{Cl}$ has been populated via the $+1 p+2 n$ channel in the ${ }^{36} \mathrm{~S}+{ }^{208} \mathrm{~Pb}$ reaction. The corresponding $\gamma$ spectrum is very similar to the one presented here (middle panel of Fig. 2). The two spectra have very similar distributions of strength over different states, even if we observed a somewhat stronger population of the states with the single-particle character. This is in agreement with a direct mechanism where only one proton was stripped from ${ }^{40} \mathrm{Ar}$ in our case, thus enhancing the population of the states of single-particle nature.

Concerning the negative parity states, we attributed $E_{\gamma}=$ $1176.4,874.4,484.5$, and $396.5 \mathrm{keV}$ to the de-excitation of the new $11 / 2^{-}$, and of the $9 / 2^{-}, 7 / 2^{-}$, and $5 / 2^{-}$states respectively, as plotted in Fig. 5. The state at $E^{*}=2571.7 \mathrm{keV}$ is labeled as $(5 / 2,7 / 2,9 / 2)^{-}$in Ref. [38], while we have preference for $J^{\pi}=9 / 2^{-}$, the $9 / 2^{-}$state being a yrast state. The $E_{\gamma}=1176.4 \mathrm{keV}$ was attributed to the decay of the $11 / 2^{-}$
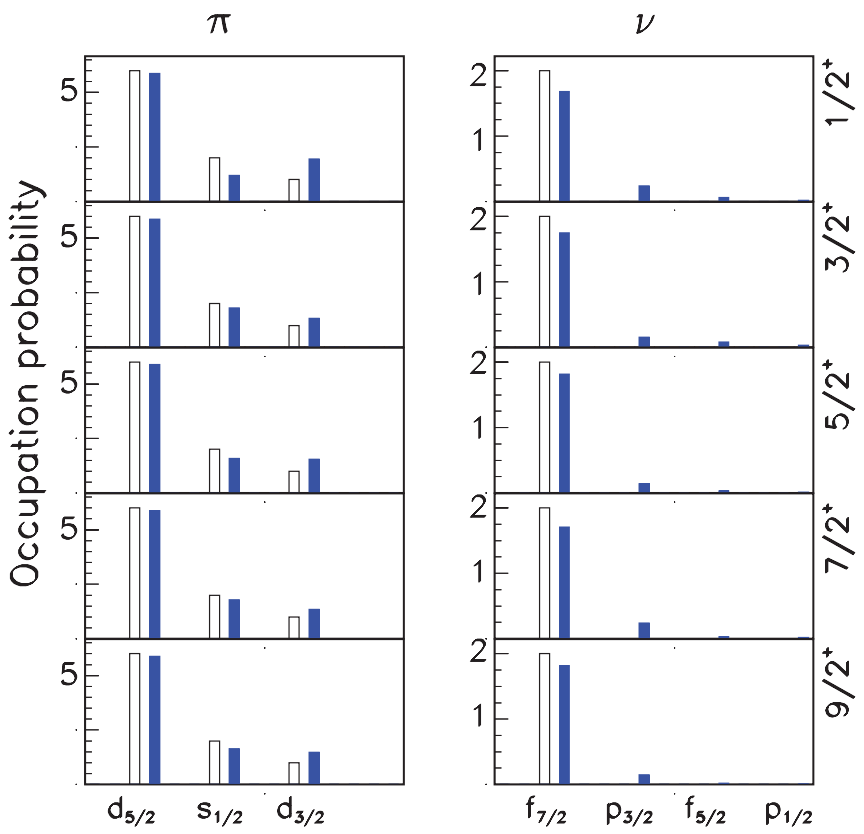

FIG. 6. (Color online) Proton (left panels) and neutron (right panels) occupation probabilities for different orbitals in ${ }^{39} \mathrm{Cl}$ (shaded bars) from the SM (SDPF-U) calculations. The states to which these probabilities correspond are labeled at the far right. Empty bars correspond to the $\pi\left(d_{5 / 2}\right)^{6}\left(s_{1 / 2}\right)^{2}\left(d_{3 / 2}\right)^{1} \otimes v\left(f_{7 / 2}\right)^{2}$ pure configuration.

state, following its coincidence with the 484.5 and $1301.3 \mathrm{keV}$ lines, as reported in Ref. [47].

The agreement between the calculated (SM using the SDPF-U interaction) and measured energies is, in general, very good (see Fig. 5). This is particularly clear for the higher spin states, $11 / 2^{+}$and $15 / 2^{+}$, where the coherent contribution of many wave-function components is expected to be less important.

According to SM calculations, for the $3 / 2_{\text {g.s. }}^{+}$about $\sim 75 \%$ of the squared amplitudes of the wave function are with two neutrons in $f_{7 / 2}$, of which $66 \%$ have $\pi\left(s_{1 / 2}\right)^{2}\left(d_{3 / 2}\right)^{1}$ and $8 \%$ have $\pi\left(s_{1 / 2}\right)^{1}\left(d_{3 / 2}\right)^{2}$ proton configurations. Two neutrons promoted to the $p_{3 / 2}$ contribute with about $4 \%$. The configuration of the $1 / 2_{1}^{+}$state is with two neutrons in $f_{7 / 2}$ $(67 \%)$ and one neutron promoted to the $p_{3 / 2}$ orbital $(11 \%)$. For the proton configuration, the dominant one is with one proton in the $s_{1 / 2}$ orbital $(\sim 62 \%)$, as one would expect for states of single-particle nature.

The configurations with the two neutrons in $f_{7 / 2}$ and the protons either in $d_{3 / 2}(51 \%)$ or in $s_{1 / 2}(23 \%)$ contribute to the squared amplitude of the $5 / 2_{1}^{+}$state with $\sim 75 \%$. A similar behavior is present in the higher spin states, $7 / 2^{+}, 9 / 2^{+}$, and $11 / 2^{+}$, where the configuration with the two neutrons in $f_{7 / 2}$ and one proton in $d_{3 / 2}$ contributes in between $\sim 50 \%$ and $\sim 70 \%$. For the $7 / 2^{+}, 9 / 2^{+}$, and $11 / 2^{+}$states the promotion of one neutron to the $p_{3 / 2}$ orbital starts to be important. In order to produce the $15 / 2^{+}$spin the neutron has to be promoted to the $f_{5 / 2}$ orbital. To illustrate the importance of the different proton and neutron orbitals discussed above, we depict in Fig. 6 the occupation probabilities of selected populated states (from 
TABLE I. The $\gamma$ transitions assigned in the present work to the ${ }^{39,41,43} \mathrm{Cl}$ isotopes. In the third column, the transition intensities (in effective number of counts) after taking into account the Clara efficiency, are listed. The label $*$ indicates new $\gamma$ transitions identified in the present work, or some of the known transitions or levels according to Ref. [38] to which additional information has been added. The $E_{\gamma}=682.8 \mathrm{keV}$ (and accordingly the $E_{i}$ ) in ${ }^{39} \mathrm{Cl}$ slightly differs from the value given in [38] where it was quoted without error.

\begin{tabular}{cccccc}
\hline \hline Isotope & $E_{\gamma}(\mathrm{keV})$ & $I_{\gamma}$ & $E_{i}(\mathrm{keV})$ & $J_{i}^{\pi}$ & $J_{f}^{\pi}$ \\
\hline${ }^{39} \mathrm{Cl}$ & $396.4(1)$ & $1300(90)$ & 396.4 & $1 / 2^{+}$ & $3 / 2^{+}$ \\
& $396.5(1)$ & $30(30)$ & 1697.3 & $5 / 2^{-}$ & $\left(5 / 2^{+}\right)$ \\
& $410.6(4)$ & $240(30)$ & 2834.4 & $\left(11 / 2^{+}\right)$ & $\left(9 / 2^{+}\right)$ \\
& $443.7(3)$ & $140(20)$ & 1745.0 & $\left(7 / 2^{+}\right)$ & $\left(5 / 2^{+}\right)$ \\
& $484.5(4)$ & $630(30)$ & 1786.0 & $\left(7 / 2^{-}\right)$ & $\left(5 / 2^{+}\right)$ \\
& $637.8(7)$ & $450(90)$ & 2423.7 & $\left(9 / 2^{+}\right)$ & $\left(7 / 2^{-}\right)$ \\
& $682.8(8)^{*}$ & $300(50)$ & $3517.2(8)$ & $\left(15 / 2^{+}\right)$ & $\left(11 / 2^{+}\right)$ \\
& $874.4(5)$ & $50(20)$ & 2571.7 & $\left(9 / 2^{-}\right)$ & $5 / 2^{-}$ \\
& $1065(1)^{*}$ & $50(30)$ & & & \\
& $1089(1)$ & $160(30)$ & 2834.4 & $\left(11 / 2^{+}\right)$ & $\left(7 / 2^{+}\right)$ \\
& $1123(1)$ & $170(40)$ & 2423.7 & $\left(9 / 2^{+}\right)$ & $\left(5 / 2^{+}\right)$ \\
& $1176.4(5)^{*}$ & $210(30)$ & $2962.4(5)$ & $\left(11 / 2^{-}\right)$ & $\left(7 / 2^{-}\right)$ \\
& $1301.3(3)$ & $1300(90)$ & 1301.2 & $\left(5 / 2^{+}\right)$ & $3 / 2^{+}$ \\
& $1326(2)$ & $120(30)$ & 1722.5 & $5 / 2^{+}$ & $1 / 2^{+}$ \\
& $1722(2)$ & $150(30)$ & 1722.5 & $5 / 2^{+}$ & $3 / 2^{+}$ \\
& $1744.5(8)$ & $430(60)$ & 1745.0 & $\left(7 / 2^{+}\right)$ & $3 / 2^{+}$ \\
& $1840.5(7)$ & $130(20)$ & 2237.9 & $1 / 2^{+}$ & $1 / 2^{+}$ \\
& $2060.0(9)$ & $190(30)$ & 2060.4 & $5 / 2^{+}$ & $3 / 2^{+}$ \\
${ }^{43} \mathrm{Cl} \mathrm{Cl}$ & $129.7(1)^{*}$ & $190(20)$ & 130.2 & $\left(3 / 2^{+}\right)$ & $\left(1 / 2^{+}\right)$ \\
& $506.3(4)^{*}$ & $50(10)$ & $2718(2)$ & $\left(9 / 2^{-}, 11 / 2^{-}\right)$ & $\left(9 / 2^{+}\right)$ \\
& $553.7(4)$ & $120(20)$ & 1444.8 & $\left(7 / 2^{+}\right)$ & $\left(5 / 2^{+}\right)$ \\
& $583.5(2)^{*}$ & $100(10)$ & $1475.3(8)$ & $\left(5 / 2^{-}, 7 / 2^{-}\right)$ & $\left(5 / 2^{+}\right)$ \\
& $735.1(3)^{*}$ & $100(10)$ & $2210.4(8)$ & $\left(9 / 2^{+}\right)$ & $\left(7 / 2^{-}\right)$ \\
& $761.7(2)$ & $270(20)$ & $891.4(2)$ & $\left(5 / 2^{+}\right)$ & $\left(3 / 2^{+}\right)$ \\
& $1006.1(8)$ & $100(20)$ & $2451.2(8)$ & $\left(11 / 2^{+}\right)$ & $\left(7 / 2^{+}\right)$ \\
& $1244(1)^{*}$ & $60(20)$ & $2718(2)$ & $\left(9 / 2^{-}, 11 / 2^{-}\right)$ & $\left(5 / 2^{-}, 7 / 2^{-}\right)$ \\
& $1346(1)^{*}$ & $240(80)$ & $1475.3(8)$ & $\left(5 / 2^{-}, 7 / 2^{-}\right)$ & $\left(3 / 2^{+}\right)$ \\
& & $3(1)$ & $329(4)$ & $\left(3 / 2^{+}\right)$ & $\left(1 / 2^{+}\right)$ \\
\hline \hline
\end{tabular}

$1 / 2^{+}$to $\left.9 / 2^{+}\right)$. The $\mathrm{SM}$ calculated occupation probabilities are compared with the expected $\pi\left(d_{5 / 2}\right)^{6}\left(s_{1 / 2}\right)^{2}\left(d_{3 / 2}\right)^{1} \otimes v\left(f_{7 / 2}\right)^{2}$ configuration. In general, in comparison with the ${ }^{37} \mathrm{Cl}$ isotope, where the main component of the squared amplitudes of wave functions of $1 / 2^{+}, 3 / 2^{+}, 5 / 2^{+}$and $7 / 2^{+}$contributes with more than $90 \%$, for the ${ }^{39} \mathrm{Cl}$ nucleus the same (main) component is reduced to $\sim 60 \%$. At the same time, the decay pattern becomes more complicated and the transitions between the positive and negative parity states much stronger.

When moving toward more neutron-rich $\mathrm{Cl}$ isotopes, spectroscopic information becomes more and more scarce. This fact is closely connected with the difficulty of accessing this region of the nuclear chart. The knowledge of the level scheme of ${ }^{41} \mathrm{Cl}$ is limited to the three lowest lying states from the study of the ${ }^{37} \mathrm{Cl}+{ }^{160} \mathrm{Gd}$ deep-inelastic collision [50]. The 129.7 , and $761.7 \mathrm{keV}$ transitions were identified to deexcite the two lowest excited states, $\left(3 / 2^{+}\right)$and $\left(5 / 2^{+}\right)$, respectively. A $\left(1 / 2^{+}\right)$assigment was adopted for the ground state of ${ }^{41} \mathrm{Cl}[50]$. This is in agreement with the direct feeding to the $1 / 2^{+}$states

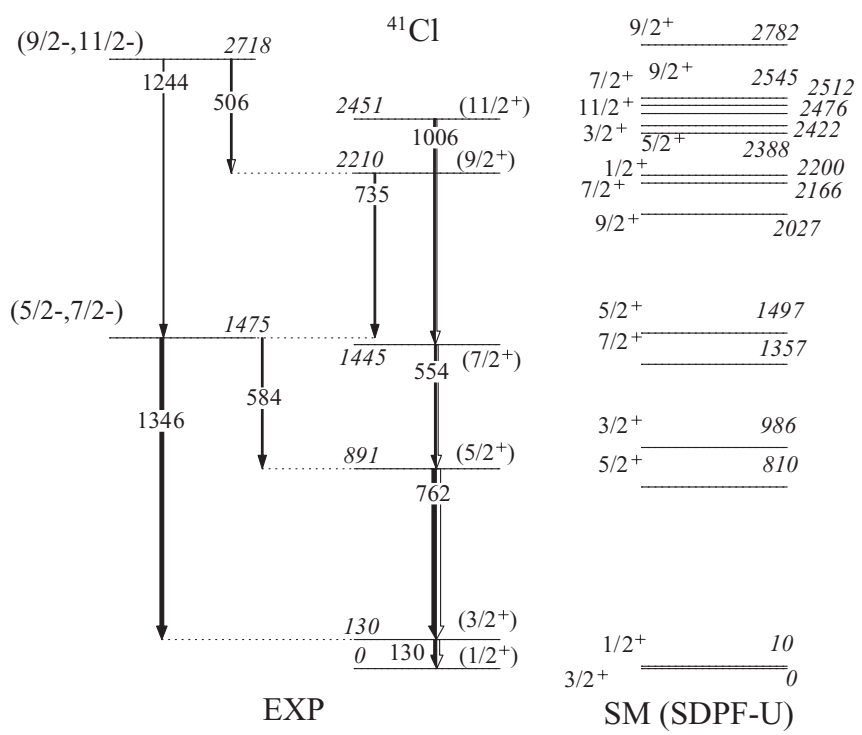

FIG. 7. Experimental negative and positive parity (left) and SM calculated (right) level scheme of ${ }^{41} \mathrm{Cl}$, populated via the $-1 p+2 n$ channel. Relative $\gamma$-ray intensities (left) are indicated by the widths of the arrows. Only the levels populated in our measurement are plotted. The energy, spin and parity of levels, their branching ratios (with tabulated $\gamma$ intensity $I_{\gamma} \geqslant 10$ ), and energy of transitions, where known, are as in Ref. [38]. SM calculations have been done by using the SDPF-U interaction in the $0 \hbar \omega$ space, therefore only the positive parity states are described and they are plotted with energies up to $3000 \mathrm{keV}$ (right).

in ${ }^{41} \mathrm{Ar}$ observed in the allowed $\beta$ decay of ${ }^{41} \mathrm{Cl}$ [51], although the $3 / 2^{+}$assignment cannot be completely ruled out. The assumption that $1 / 2^{+}$is the ground state agrees well with our observation of a unique $129.7 \mathrm{keV}$ transition populating the ${ }^{41} \mathrm{Cl}$ ground state. The $E 2$ transition deexciting the $5 / 2^{+}$ to the $1 / 2^{+}$is absent in both ${ }^{37} \mathrm{Cl}$ and ${ }^{39} \mathrm{Cl}$ isotopes. Therefore, as we observed only the single cascade structure in ${ }^{41} \mathrm{Cl}$, our level scheme is built on the assumption that the ground state of ${ }^{41} \mathrm{Cl}$ is $1 / 2^{+}$.

The SM calculations for chlorine isotopes predict that the $3 / 2^{+}$and $1 / 2^{+}$levels come closer in energy, and eventually cross as the neutron number increases. According to the SDPF$\mathrm{U}$ interaction this crossover falls in ${ }^{43} \mathrm{Cl}$. The experimental excitation energy of the $3 / 2^{+}$states in ${ }^{43} \mathrm{Cl}$ is $E^{*}=329 \mathrm{keV}$ [8], thus more than twice with respect to the almost degenerate ${ }^{41} \mathrm{Cl}$ case $(E=129.7 \mathrm{keV})$, indicating that the crossover has already occurred. These aspects will be more extensively discussed in Sec. IV.

Most of the available information about ${ }^{41} \mathrm{Cl}$ is coming from the ${ }^{37} \mathrm{Cl}+{ }^{160} \mathrm{Gd}$ measurement [50], where the transitions $\sim 130$ and $\sim 761 \mathrm{keV}$ have been identified, and from the ${ }^{36} \mathrm{~S}+{ }^{176} \mathrm{Yb}$ measurement [52] where two additional transitions $\sim 554$ and $\sim 1006 \mathrm{keV}$ have been reported. In the present work $\sim 10$ transitions were uniquely attributed to ${ }^{41} \mathrm{Cl}$ (see Table I) including the four previously known transitions. The transitions observed here agree with the results of the ${ }^{36} \mathrm{~S}+{ }^{208} \mathrm{~Pb}$ Prisma-Clara measurement [45]. In this last work, additional information has been extracted by a further analysis of the ${ }^{37} \mathrm{Cl}+{ }^{160} \mathrm{Gd} \gamma$-coincident data [47]. 
Since $\gamma-\gamma$ coincidence data were not available in our study, the new $\gamma$ rays observed in coincidence with ${ }^{41} \mathrm{Cl}$ were placed in the level scheme (shown in Fig. 7) on the basis of energy matching and intensity arguments and with the help of $\gamma-\gamma$ coincidence data from the work of [45-47]. For the spin assignments we took profit of the comparison with the ${ }^{39} \mathrm{Cl}$ isotope, where the transfer flux was distributed over both the negative and positive parity bands. It is evident in the ${ }^{39} \mathrm{Cl}$ level scheme that the populated states with the same spin but different parity appear at a similar energy. In our opinion the same occurs in ${ }^{41} \mathrm{Cl}$. Using these arguments, in Fig. 7 we assigned spin-parity $\left(11 / 2^{+}\right)$to the state at $E^{*}=2451.2 \mathrm{keV}$, at variance with Ref. [52]. We also have added a new state with spin $\left(9 / 2^{+}\right)$at $E^{*}=2210.4 \mathrm{keV}$ in the positive parity band. This assignment is in good agreement with the SM calculations.

Similarly to ${ }^{39} \mathrm{Cl}$, we expect to populate the negative parity states also for ${ }^{41} \mathrm{Cl}$. In the negative parity band of ${ }^{39} \mathrm{Cl}$, we observed the decay of $5 / 2^{-},\left(7 / 2^{-}\right),\left(9 / 2^{-}\right)$, and $\left(11 / 2^{-}\right)$states. The decay chain $\left(11 / 2^{-}\right) \rightarrow\left(7 / 2^{-}\right) \rightarrow 5 / 2^{+}$ was stronger than the decay chain $\left(9 / 2^{-}\right) \rightarrow 5 / 2^{-} \rightarrow 5 / 2^{+}$. Here, for ${ }^{41} \mathrm{Cl}$, we attributed the new $\gamma$ transitions (506.3, $583.5,1244$, and $1346 \mathrm{keV}$ ) to the decay of the negative parity states. Following the constructed decay scheme of ${ }^{39} \mathrm{Cl}$, we tentatively attributed the 1346 and $583.5 \mathrm{keV}$ lines to the deexcitation of the $\left(7 / 2^{-}\right)$state [even if $\left(5 / 2^{-}\right)$assignment cannot be completely excluded], and the lines 1244 and $506.3 \mathrm{keV}$ to the decay of $\left(11 / 2^{-}, 9 / 2^{-}\right)$state, respectively. As the deexcitation of the $\left(11 / 2^{-}\right)$state was stronger than that of $\left(9 / 2^{-}\right)$in ${ }^{39} \mathrm{Cl}$, our preferences for the states at 2718 and $1475.3 \mathrm{keV}$ are $\left(11 / 2^{-}\right)$and $\left(7 / 2^{-}\right)$, respectively. In this way the doublets of the same spin and different parity behave very similarly as in the experimentally better known isotopes ${ }^{39} \mathrm{Cl}$ and ${ }^{37} \mathrm{Cl}$.

Looking more closely at the wave functions of the populated states we can see that the identified positive parity states consist of strong mixing of the $\pi\left(s_{1 / 2}\right)^{1}\left(d_{3 / 2}\right)^{2}$ and $\pi\left(s_{1 / 2}\right)^{2}\left(d_{3 / 2}\right)^{1}$ shell configurations with the valence neutrons in the $f_{7 / 2}$ orbital. For the $5 / 2^{+}$and $9 / 2^{+}$states configurations involving the unpaired proton in $s_{1 / 2}$ is dominant $(\sim 40 \%)$, while for $3 / 2^{+}$and $7 / 2^{+}$ the dominant configuration has the unpaired proton in $d_{3 / 2}$ (also $\sim 40 \%$ ). To describe these states, a relatively large (up to $\sim 20 \%$ ) contribution from the configurations involving the promotion of one or two neutrons to the $p_{3 / 2}$ orbital is also required.

The main components of the wave functions of the negative parity states in ${ }^{37} \mathrm{Cl}$ are connected, as already discussed, with the promotion of one proton or one neutron from the $d_{3 / 2}$ and $s_{1 / 2}$ to the $f_{7 / 2}$ orbitals. Being the states rather pure with usually one dominant wave function component, the transitions between the positive and negative parity states turn out to be weak. Here, for ${ }^{41} \mathrm{Cl}$, where the main component of the wave function contributes at most with $\sim 40 \%$ to the squared amplitude, the interband transitions become more important.

The ${ }^{43} \mathrm{Cl}$ nucleus was very weakly populated in our measurement, and in the fragment $-\gamma$ coincidence spectra about $\sim 150$ events were collected over the whole energy range (up to $4 \mathrm{MeV}$ ). This number of events is at the limit of the sensitivity of the performed measurement. The only $\gamma$ transition which could be attributed to ${ }^{43} \mathrm{Cl}$ is $E_{\gamma}=328 \mathrm{keV}$, which corresponds to the transition between the $\left(3 / 2^{+}\right)$and $\left(1 / 2_{\text {g.s. }}^{+}\right)$states [38].

\section{B. Structure of odd-odd $\mathrm{Cl}$ isotopes}

Experimentally, odd-odd nuclei are more difficult to study since the presence of a large number of states at low energy results in the spread of the deexcitation flux over several transitions, reducing the corresponding intensities. Significant progress has been achieved by using $\gamma$-fragment coincidence techniques, where $\gamma$ transitions could be attributed to specific isotopes. This method turned out to be particularly powerful when combined with complementary studies performed via $\gamma-\gamma$ coincidences, essential in building level schemes. Newly identified $\gamma$ transitions for the odd-odd $\mathrm{Cl}$ isotopes are presented below. When available, we used information from $\gamma-\gamma$ coincidence studies.

Spectra of $\gamma$ rays detected in coincidence with the odd-odd $\mathrm{Cl}$ isotopes are depicted in Fig. 3, while their level schemes are presented in Figs. 8, 9, and 10 for ${ }^{38} \mathrm{Cl},{ }^{40} \mathrm{Cl}$, and ${ }^{42} \mathrm{Cl}$, respectively. The $\gamma$ transitions are listed in Table II.

The ${ }^{38} \mathrm{Cl}$ nucleus [38] was extensively studied in $(d, p)$ reactions, in particle- $\gamma$ coincident measurements, and via $\beta$ decay measurements. These experiments provided states up to $\sim 8 \mathrm{MeV}$ with spin assignments up to $J \leqslant 5$. Recently, ${ }^{38} \mathrm{Cl}$ was studied in heavy ion induced binary reactions and the level scheme was extended up to higher spin states $[45,53]$. The

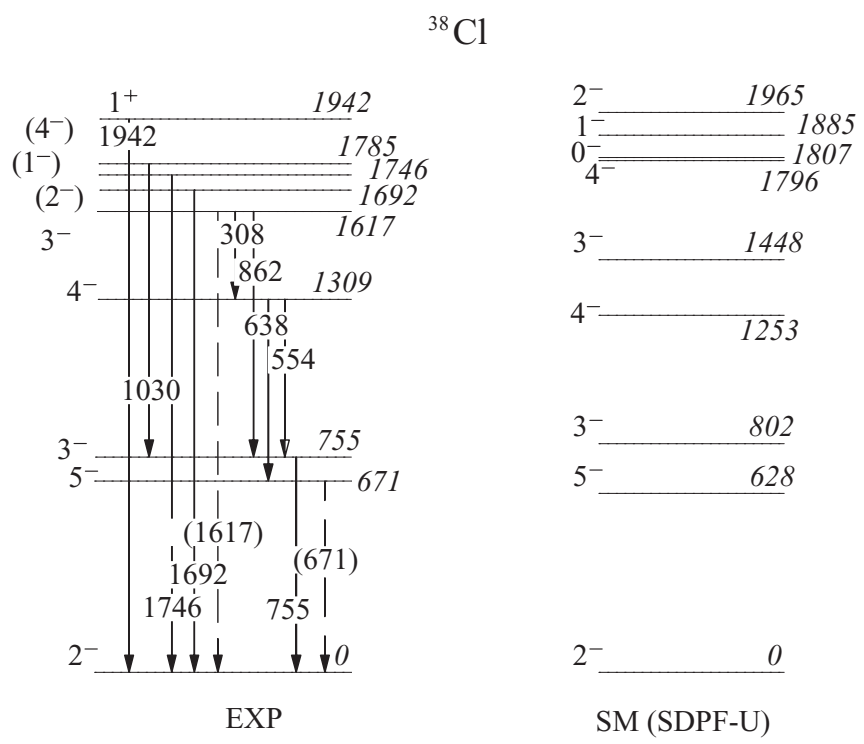

FIG. 8. Experimental (left) and SM calculated (right) level scheme of ${ }^{38} \mathrm{Cl}$, populated via the $-1 p-1 n$ channel. Relative $\gamma$-ray intensities (left) are indicated by the widths of the arrows (see text for dashed line). Only the levels populated in our measurement are plotted. The energy, spin and parity of levels, their branching ratios (with tabulated $\gamma$ intensity $I_{\gamma} \geqslant 10$ ), and energy of transitions, where known, are as in Ref. [38]. SM calculations have been done by using the SDPF-U interaction in the $0 \hbar \omega$ space, therefore only the negative parity states are described and they are plotted with energies up to $\sim 2000 \mathrm{keV}$ (right). 


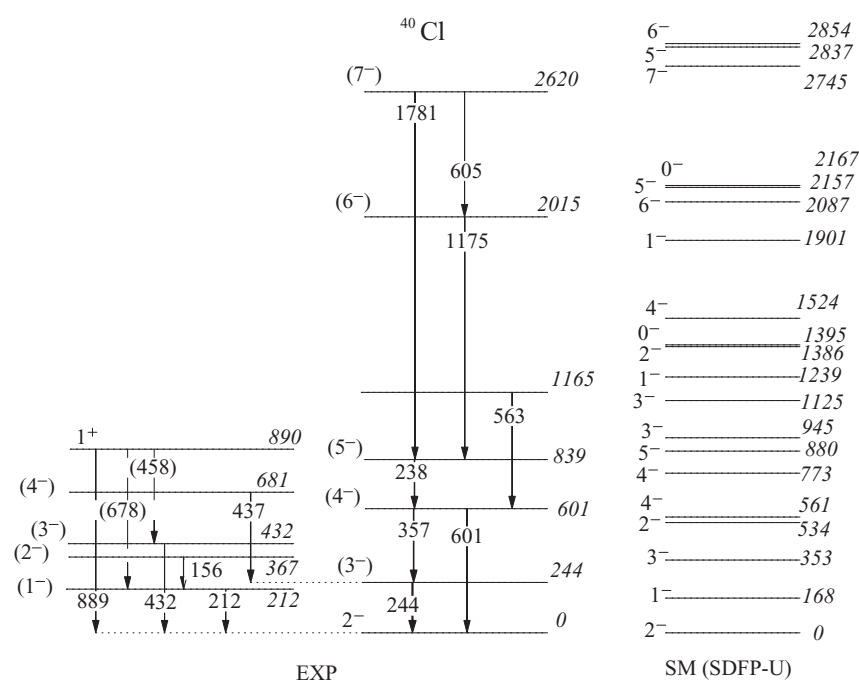

FIG. 9. Experimental [separated in two bands for clarity's sake] (left), and SM calculated (right) level scheme of ${ }^{40} \mathrm{Cl}$, populated via the $-1 p+1 n$ channel. Relative $\gamma$-ray intensities (left) are indicated by the widths of the arrows. Only the states observed in our measurement are plotted. The energy, spin and parity of levels, their branching ratios (with tabulated $\gamma$ intensity $I_{\gamma} \geqslant 10$ ), and energy of transitions, where known, are as in Ref. [38]. Some of the weaker intensity $\gamma$ rays were not observed in our measurement with sufficient statistical accuracy, thus they are plotted as dashed lines. SM calculations have been done by using the SDPF-U interaction in the $0 \hbar \omega$ space, therefore only the negative parity states are described and they are plotted with energies up to $\sim 2800 \mathrm{keV}$ (right).

level scheme presented in Fig. 8 is in good agreement with the results of Ref. [53]. In our measurements we could partially confirm their results, although the accumulated statistics for the high-energy $\gamma$ transitions (see dashed arrows in Fig. 8) were not sufficient to add significant new information. The transition $E_{\gamma}=671 \mathrm{keV}$ was not observed in the present experiment since, due to its isomeric nature $\left(T_{1 / 2}=715 \mathrm{~ms}\right)$, most of the produced ${ }^{38} \mathrm{Cl}$ ions fly away before its detection.
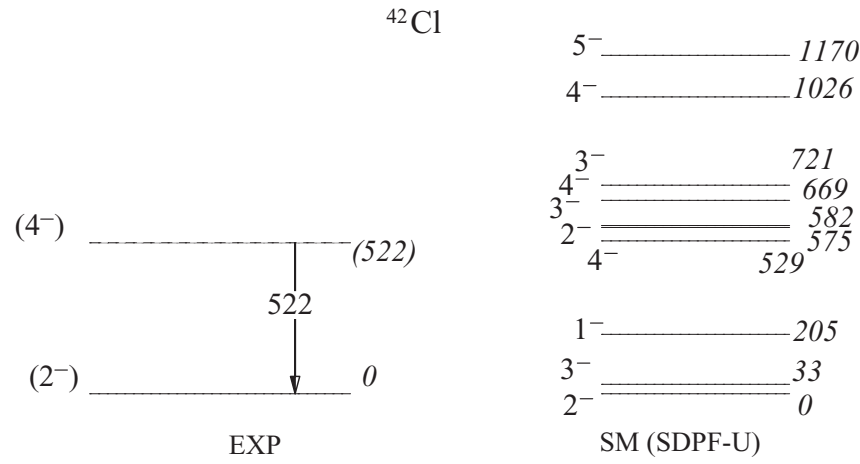

FIG. 10. Experimental (left) and SM calculated (right) level scheme of ${ }^{42} \mathrm{Cl}$, populated via the $-1 p+3 n$ channel. SM calculations have been done by using the SDPF-U interaction in the $0 \hbar \omega$ space, therefore only the negative parity states are described and they are plotted with energies up to $\sim 1200 \mathrm{keV}$ (right).
TABLE II. The $\gamma$ transitions assigned in the present work to the ${ }^{38,40,42} \mathrm{Cl}$ isotopes. The known transitions and levels are as in Ref. [38]. In the third column, the transition intensities (in effective number of counts) after taking into account the Clara efficiency, are listed. The * label indicates new $E_{\gamma}$ transitions identified in the present work, or some of the known transitions or levels according to Ref. [38] to which additional information has been added.

\begin{tabular}{|c|c|c|c|c|c|}
\hline Isotope & $E_{\gamma}(\mathrm{keV})$ & $I_{\gamma}$ & $E_{i}(\mathrm{keV})$ & $J_{i}^{\pi}$ & $J_{f}^{\pi}$ \\
\hline \multirow[t]{16}{*}{${ }^{38} \mathrm{Cl}$} & $169(1)^{*}$ & $150(30)$ & & & \\
\hline & $290.8(4)^{*}$ & $80(20)$ & & & \\
\hline & $308.4(6)$ & $50(10)$ & 1617.4 & $3^{-}$ & $4^{-}$ \\
\hline & $553.6(6)$ & $54(8)$ & 1309.1 & $4^{-}$ & $3^{-}$ \\
\hline & $638.0(7)$ & $230(40)$ & 1309.1 & $4^{-}$ & $5^{-}$ \\
\hline & $755.0(3)$ & $300(40)$ & 755.4 & $3^{-}$ & $2^{-}$ \\
\hline & $862.0(3)$ & $70(20)$ & 1617.4 & $3^{-}$ & $3^{-}$ \\
\hline & $1029.6(9)$ & $110(20)$ & 1784.97 & $\left(4^{-}\right)$ & $3^{-}$ \\
\hline & $1063(1)^{*}$ & $40(20)$ & & & \\
\hline & $1691.8(7)$ & $60(20)$ & 1692.46 & $\left(2^{-}\right)$ & $2^{-}$ \\
\hline & $1746(3)$ & $50(15)$ & 1745.8 & $\left(1^{-}\right)$ & $2^{-}$ \\
\hline & 1942(3) & $100(30)$ & 1942.0 & $1^{+}$ & $2^{-}$ \\
\hline & $2631(2)^{*}$ & $40(10)$ & & & \\
\hline & $2677(3)^{*}$ & $40(15)$ & & & \\
\hline & $2758(3)^{*}$ & $40(15)$ & & & \\
\hline & $2971(5)^{*}$ & $20(15)$ & & & \\
\hline \multirow[t]{15}{*}{${ }^{40} \mathrm{Cl}$} & $156(1)$ & $100(20)$ & 367.1 & $\left(2^{-}\right)$ & $\left(1^{-}\right)$ \\
\hline & $211.6(4)$ & $80(20)$ & 211.6 & $\left(1^{-}\right)$ & $2^{-}$ \\
\hline & $219.0(4)$ & $90(20)$ & & & \\
\hline & 237.9(1) & $310(30)$ & 839.2 & $\left(5^{-}\right)$ & $\left(4^{-}\right)$ \\
\hline & $244.0(1)$ & $780(40)$ & 244.0 & $\left(3^{-}\right)$ & $2^{-}$ \\
\hline & $357.4(2)$ & $380(60)$ & 601.3 & $\left(4^{-}\right)$ & $\left(3^{-}\right)$ \\
\hline & $431.8(4)$ & $130(50)$ & 431.8 & $\left(0^{-}\right)$ & $2^{-}$ \\
\hline & $436.9(1)$ & $290(50)$ & 680.95 & $\left(4^{-}\right)$ & $\left(3^{-}\right)$ \\
\hline & $563.3(4)^{*}$ & $170(20)$ & $1164.7(4)$ & & $\left(4^{-}\right)$ \\
\hline & $601.1(1)$ & $38(9)$ & 601.3 & $\left(4^{-}\right)$ & $2^{-}$ \\
\hline & $605(2)$ & $30(8)$ & 2620.4 & $\left(7^{-}\right)$ & $\left(6^{-}\right)$ \\
\hline & $889.2(5)$ & $40(20)$ & 889.5 & $1^{+}$ & $2^{-}$ \\
\hline & $1175(1)$ & $110(30)$ & 2014.7 & $\left(6^{-}\right)$ & $\left(5^{-}\right)$ \\
\hline & $1360(1)^{*}$ & $65(20)$ & & & \\
\hline & 1781(1) & $50(20)$ & 2620.4 & $\left(7^{-}\right)$ & $\left(5^{-}\right)$ \\
\hline${ }^{42} \mathrm{Cl}$ & $522(1)^{*}$ & $20(4)$ & 520.5 & $\left(4^{-}\right)$ & $\left(2^{-}\right)$ \\
\hline
\end{tabular}

The strongest populated states at low excitation energy are those with spin-parity $5^{-}, 3^{-}$, and $4^{-}$, which, together with the $2^{-}$ground state, form in a very simple picture a quadruplet of levels built on the $\pi\left(d_{3 / 2}\right) \otimes v\left(f_{7 / 2}\right)$ configuration. SM calculated (right part of Fig. 8) energies agree well with the experimental ones. Looking in more detail in the SM calculated wave functions we can infer that about more than $80 \%$ of the squared amplitudes of the ground and $5^{-}$states are, in fact, in this simple configuration, an uncoupled proton in the $d_{3 / 2}$ and an uncoupled neutron in the $f_{7 / 2}$ orbitals. The $3^{-}$and $4^{-}$ states are more mixed, and proton configuration $\pi\left(s_{1 / 2}\right)^{1}\left(d_{3 / 2}\right)^{2}$ or $\pi\left(s_{1 / 2}\right)^{2}\left(d_{3 / 2}\right)^{1}$ with the neutron either in the $f_{7 / 2}$ or in the $p_{3 / 2}$ orbitals contribute significantly.

In our measurement we observed also the transition which depopulates the $3_{2}^{-}$state at $1617.4 \mathrm{keV}$. The SM calculations assign to this state a rather complex wave function; i.e., the 
configurations $\pi\left(s_{1 / 2}\right)^{2}\left(d_{3 / 2}\right)^{1} \otimes v\left(p_{3 / 2}\right)^{1}$ and $\pi\left(s_{1 / 2}\right)^{1}\left(d_{3 / 2}\right)^{2}$ $\otimes v\left(f_{7 / 2}\right)^{1}$ contribute with a similar strength $(\sim 40 \%$ each). For the state at $1745.8 \mathrm{keV}$, identified as $0^{-}, 1^{-}$[38] and for the state at $1784.97 \mathrm{keV}$, identified as $2^{-}, 3^{-}, 4^{-}$[38], the SM calculations have preference for spins $1^{-}$and $4^{-}$, as indicated in Fig. 8. Besides these negative parity states, we also observed the transitions from the $1^{+}$state at $1942 \mathrm{keV}$. In addition, we observed other transitions, uniquely attributed to ${ }^{38} \mathrm{Cl}$, which we could not place in the level scheme of Fig. 8. All these transition are listed in Table II. We observed that the two very strong transitions at $E_{\gamma}=169$ and $290.8 \mathrm{keV}$ are in coincidence with each other. We also observed a clear peak at $1063 \mathrm{keV}$, though with low statistics. In the high-energy part of the $\gamma$ spectrum of ${ }^{38} \mathrm{Cl}$ there are some indications of the high-energy $\gamma$ rays at 2631, 2677, 2758, and $2971 \mathrm{keV}$.

The $\gamma$-ray spectrum in coincidence with ${ }^{40} \mathrm{Cl}$ is depicted in the middle panel of Fig. 3, while the corresponding level scheme is shown in Fig. 9. The $\gamma$-ray transitions are listed in Table II. Previous information on ${ }^{40} \mathrm{Cl}$ was obtained from $\beta$-decay [54] and heavy ion fusion studies [55,56]. The same nucleus was also populated in the "thick" -target $\gamma-\gamma$ coincidence measurements [46,47].

Similar to the ${ }^{38} \mathrm{Cl}$ case, it is expected that the $\pi\left(s_{1 / 2}\right)^{2}\left(d_{3 / 2}\right)^{1} \otimes v\left(f_{7 / 2}\right)^{3}$ configuration dominates the wave functions of the low-lying states. Here, the expected multiplet, $5^{-}, 4^{-}, 3^{-}$and $2^{-}$, according to the SM calculations, has a higher configuration mixing in the wave functions compared with the ${ }^{38} \mathrm{Cl}$ case. The configuration with the uncoupled $d_{3 / 2}$ proton and $f_{7 / 2}$ neutron contributes to the squared amplitudes with $\sim 50 \%$ for the $5^{-}, 4^{-}$, and $2^{-}$states, and $\sim 40 \%$ for the $3^{-}$state. For these states the configuration with an uncoupled neutron in $p_{3 / 2}$ is also important $(\sim 5 \%-15 \%)$. It is interesting to point out that in the low excitation energy region (up to the $1^{+}$state at $889.5 \mathrm{keV}$ ) we observed deexcitations from all known states of ${ }^{40} \mathrm{Cl}$ [38]. Comparing this excitation energy region with the SM calculations we can conclude that all states up to $1 \mathrm{MeV}$ have their experimental "partner", except the $2_{2}^{-}$ at $E^{*}=534 \mathrm{keV}$, and the $3_{2}^{-}$at $945 \mathrm{keV}$ non-yrast states. From the experimental side, the state at $E^{*}=367.1 \mathrm{keV}$ is labeled as (2) and the state at $431.8 \mathrm{keV}$ as $\left(0^{-}: 3^{+}\right)$in Ref. [38]. Thus, we assumed that the observed state at $367.1 \mathrm{keV}$ corresponds to the "missing" SM calculated $2_{2}^{-}$at $534 \mathrm{keV}$, even if the predicted excitation energy is somewhat higher. The state at $431.8 \mathrm{keV}$ could correspond to the SM calculated $33_{2}^{-}$state (as it is labeled in Fig. 9), or it could be a member of the positive parity band. We would also like to point out a remarkable good agreement, within $\sim 100 \mathrm{keV}$, between the calculated and measured $6^{-}$and $7^{-}$excitation energies. These relatively high spin states, even if separated in energy by more than $1 \mathrm{MeV}$ from the low-lying states, are rather strongly populated by the transfer mechanism. In addition, we observed 219.0 and $563.3 \mathrm{keV}$ transitions. The $219.0 \mathrm{keV}$ transition was reported in the previous studies [45-47,55,56]. However, as its placement in the level scheme has been controversial, the transition has been listed in Table II but it was not placed in the level scheme in Fig. 9. From our fragment $-\gamma-\gamma$ coincident data with very low statistics for this isotope the 219.0 transition seems to be in coincidence with 436.9 and $244.0 \mathrm{keV}$, giving a state at $899.9(4) \mathrm{keV}$. The $563.3 \mathrm{keV}$ transition has few coincident fragment $-\gamma-\gamma$ events with 357.4 and $244.0 \mathrm{keV}$ transition, indicating the existence of a state at $1164.7 \mathrm{keV}$ excitation energy, as shown in Fig. 9. These states, 899.9 and $1164.7 \mathrm{keV}$, probably correspond to the SM calculated states between 1.0 and $1.6 \mathrm{MeV}$, most probably the $3^{-}$at $1125 \mathrm{keV}$, and $4^{-}$at $1524 \mathrm{keV}$.

The $\gamma$-ray spectrum in coincidence with the weakly populated ${ }^{42} \mathrm{Cl}$ isotope is plotted in Fig. 3 (bottom panel). The strongest line in the spectrum is $E_{\gamma}=522 \mathrm{keV}$. Besides this, there are indications of the transitions $E_{\gamma}=$ $117,230,275,400,1225 \mathrm{keV}$, although with very low statistics.

The ${ }^{42} \mathrm{Cl}$ nucleus was studied in the $\beta$ decay of ${ }^{42} \mathrm{~S}$ [57], where it was established that the $118 \mathrm{keV}$ transition corresponds to the decay of the first excited state [with $J_{\pi}=\left(2^{-}\right)$] to the ground state [recognized as $(3)^{-}$]. Several transitions were also reported in Ref. [45], and among them those at $\sim 117,522,275,400$, and $1225 \mathrm{keV}$ are in common with our findings. SM calculations predict the $2^{-}$state as a ground state, and the $3^{-}$state as the first excited state, these states being almost degenerate. The main wave function components of these states are very similar, i.e., the configuration $\pi\left(s_{1 / 2}\right)^{1}\left(d_{3 / 2}\right)^{2} \otimes v\left(f_{7 / 2}\right)^{5}$ contributes with $\sim 30 \%$, and the $\pi\left(s_{1 / 2}\right)^{2}\left(d_{3 / 2}\right)^{1} \otimes v\left(f_{7 / 2}\right)^{5}$ configuration with $\sim 20 \%$, while the configurations $\pi\left(s_{1 / 2}\right)^{1}\left(d_{3 / 2}\right)^{2} \otimes v\left(f_{7 / 2}\right)^{4}\left(p_{3 / 2}^{1}\right)$ and $\pi\left(s_{1 / 2}\right)^{2}\left(d_{3 / 2}\right)^{2} \otimes v\left(f_{7 / 2}\right)^{4}\left(p_{3 / 2}^{1}\right)$ contribute with $\sim 10 \%$ to the squared amplitudes of these states. The $4^{-}$state has a more simple configuration, with the $\pi\left(s_{1 / 2}\right)^{1}\left(d_{3 / 2}\right)^{2} \otimes v\left(f_{7 / 2}\right)^{5}$ component which contributes $39 \%$, while all other components (with promotion of a neutron to the $p_{3 / 2}$ or rearrangement of the protons in the $s_{1 / 2}$ and $d_{3 / 2}$ orbitals) contribute with $\leqslant 10 \%$. As the states with the larger spin and less mixed configuration have been shown to be strongly fed in this transfer reaction, we attributed the strongest observed line to the decay of the state at $522 \mathrm{keV}$, recognized as $\left(4^{-}\right)$, to the ground state (for which we also kept the SM predicted spin, i.e., $2^{-}$).

\section{SOME CONSIDERATIONS ON THE CHLORINE ISOTOPIC CHAIN}

In recent years, a special interest was dedicated to the breakdown of the $N=28$ magic number, or vanishing of the proton $Z=16$ subshell gap [1-8,10,11,15-17]. As mentioned in the Introduction, the behavior of the proton $s_{1 / 2}$ and $d_{3 / 2}$ orbitals was crucial in understanding these effects. Here we will discuss how the behavior of the $s_{1 / 2}$ and $d_{3 / 2}$ proton orbitals affects the $\mathrm{Cl}(Z=17)$ isotopic chain where its three proton holes could produce enhanced mixing [8,10,23,39]. The evolution of the energy splitting between the lowest $1 / 2^{+}$and $3 / 2^{+}$states in the chlorine isotopes is illustrated in Fig. 11, where the results of the SM calculations are shown together with the experimental data. In chlorine nuclei one expects that the $s_{1 / 2}$ orbital is completely filled and that the unpaired proton occupies the $d_{3 / 2}$ orbital, naturally leading to the $3 / 2^{+}$ground state. The ${ }^{37} \mathrm{Cl}$ nucleus has the expected spin order, i.e., $3 / 2^{+}$as a ground state and $1 / 2^{+}$as a first excited state. In this nucleus the occupation probability of the $s_{1 / 2}$ and $d_{3 / 2}$ orbitals is 1.01 and 2.09 for the $1 / 2^{+}$state 


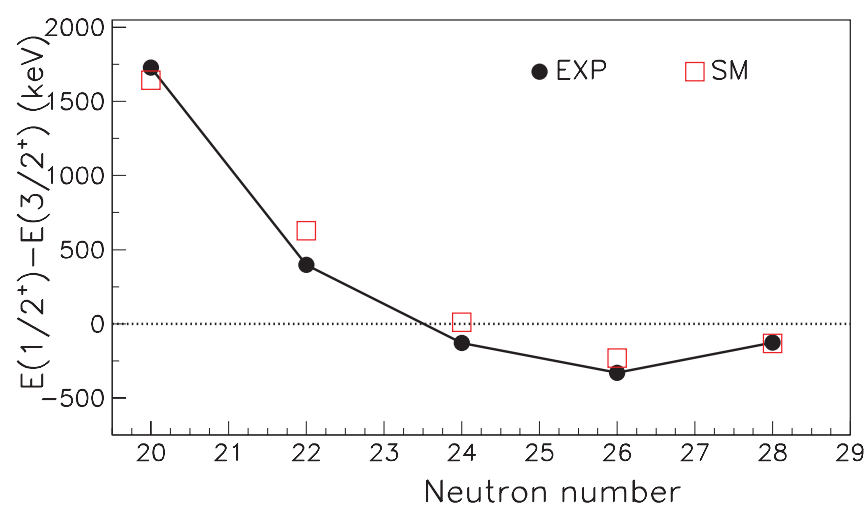

FIG. 11. (Color online) Energy splitting, as a funcion of neutron number, between the lowest $1 / 2^{+}$and $3 / 2^{+}$states in the odd-even chlorine isotopes. The experimental values are denoted with black circles while SM calculated ones are denoted with red squares.

and 1.95 and 1.11 for the $3 / 2^{+}$state. This strongly supports the single-particle picture. With increasing neutron number, the $d_{3 / 2}-s_{1 / 2}$ splitting is reduced and mixing between different proton configurations increases; at the same time, the coupling to the neutron excitation (i.e., the promotion of one or two neutrons to the $p_{3 / 2}$ ) also increases. For ${ }^{41} \mathrm{Cl}$ (middle of shell, $N=24)$ the $3 / 2^{+}$and $1 / 2^{+}$states become nearly degenerate in the SM calculations $\left(\Delta_{\mathrm{SM}} \sim 10 \mathrm{keV}\right)$ and the crossing between the two states occurs experimentally $\left(\Delta_{\mathrm{EXP}} \sim-130 \mathrm{keV}\right)$. In fact, in this case the occupation probability of the two orbits is nearly the same for the $3 / 2^{+}$state. The evolution of this splitting for $\mathrm{K}, \mathrm{Cl}$, and $\mathrm{P}$ isotopes was discussed in Ref. [39], using the same shell-model interaction, in terms of the preserved single-particle character in the isotopic chain, and of the coupling of the proton single-particle states with the neutron excitations. It was concluded that the reduced energy splitting and increased mixing of different proton configurations results in nearly quasiparticle states. In other words, the heavy $\mathrm{Cl}$ isotopes can be viewed as one proton coupled to the corresponding ${ }^{(A-1)} S$ isotope. In addition, these quasiparticles can couple to the quadrupole excitation, producing very complex wave functions. Thus, the vanishing of the $N=28$ closure in the $\mathrm{S}$ isotopes should be reflected in the $\mathrm{Cl}$ isotopic chain. This behavior can be demonstrated by inspecting the $5 / 2_{1}^{+}$states. In fact, their energies follow closely the absolute difference $\left|E\left(1 / 2^{+}\right)-E\left(3 / 2^{+}\right)\right|$as well as the energies of the $2^{+}$states of the corresponding ${ }^{\left({ }^{A-1)}\right.} \mathrm{S}$ core (see Fig. 12), except at $N=28$, where the $5 / 2^{+}$state follows more closely the $\left|E\left(1 / 2^{+}\right)-E\left(3 / 2^{+}\right)\right|$energy difference. In ${ }^{37} \mathrm{Cl}$, the main component of the $5 / 2_{1}^{+}$wave function is similar to the one of the first $1 / 2^{+}$excited state, with one proton in the $s_{1 / 2}$ orbital contributing to the squared amplitude with $93 \%$. In ${ }^{41} \mathrm{Cl}$, this proton configuration contributes only with $36 \%$. At the same time, the contributions from the promotion of a neutron to the $p_{3 / 2}$ orbital becomes more important.

Although one can hardly infer about collectivity only from information for the energy of states, especially for such complex wave functions, it is interesting to notice that the trend of the energies of the higher spin states, $7 / 2^{+}$and $9 / 2^{+}$, behave similarly as the $5 / 2^{+}$. Of course, the scarce knowledge

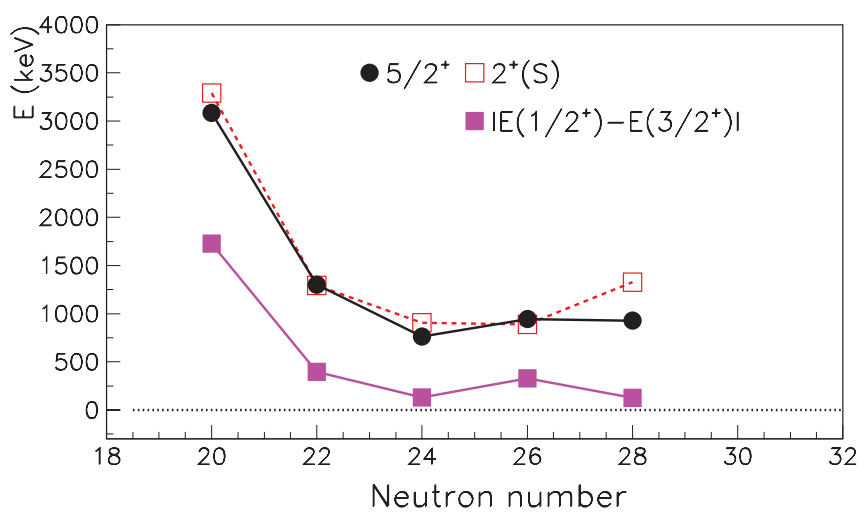

FIG. 12. (Color online) Absolute energy difference between the lowest $1 / 2^{+}$and $3 / 2^{+}$states in the odd-even chlorine isotopes (filled squares, violet) and excitation energies of the $5 / 2^{+}$states (filled circles, black), compared with the energies of the $2^{+}$states in $\mathrm{S}$ isotopes (empty squares, red), as a function of the neutron number.

about high spin states makes conclusions based on this energy systematics quite qualitative. However, even if the spin-parity assignment of the these higher states identified in the present work could not be rigorously made, the possibility to transfer large angular momenta via the transfer mechanism helps to enlighten us about the nature of the observed excitations. In addition, the SM calculations have proven to be a very good guidance in this mass and charge region. For instance, calculations turned out to agree well with experimental data in ${ }^{39} \mathrm{Cl}$ up to the energy $\sim 2.5 \mathrm{MeV}$, where we observed the decays of all adopted states. Moreover, we observed a preferential population of the yrast states in the high-energy region, as expected from the transfer mechanism. Since the same transfer mechanism would be expected to excite similar states in the less known ${ }^{41} \mathrm{Cl}$, this fact together with $\mathrm{SM}$ predictions has assisted in the assignment of $9 / 2^{+}$and $11 / 2^{+}$to the states at 2210.4 and $2451.2 \mathrm{keV}$, respectively. SM calculations predict lifetimes of these higher spin states in the $\sim 10-30$ ps range; they are therefore well suited for measurements in fragment $-\gamma$ coincidences with plunger devices [58-60].

Concerning the negative parity states, the observed excitations most likely arise from the promotion of a proton across the $Z=20$ gap. For example, in ${ }^{37} \mathrm{Cl}$ about one third of the total observed flux is within the negative parity band. All Cl isotopes being populated in the same reaction, the population of the negative parity states is highly expected also in the more neutron-rich isotopes. These arguments were taken into account in the construction of the corresponding level schemes, where we attributed some new $\gamma$ transitions to the deexcitation of the negative parity (higher spin) states in ${ }^{39} \mathrm{Cl}$ and ${ }^{41} \mathrm{Cl}$ (we remind that the negative parity states cannot be accounted for in the present shell-model space).

In the odd-odd $\mathrm{Cl}$ isotopes, the same arguments were applied. We first looked closely to the states that have been populated by the transfer mechanism in the isotopes closer to the stability line, where spectroscopic informations were available. In ${ }^{38} \mathrm{Cl}$ most of the observed flux can be attributed to the deexcitation of low-lying levels $3^{-}, 4^{-}$, and $5^{-}$. In a simple 
picture, these states can be viewed as a quadruplet of levels built on the $\pi\left(d_{3 / 2}\right) \otimes v\left(f_{7 / 2}\right)$ configuration. One would then expect to populate this quadruplet in the more neutron rich $\mathrm{Cl}$ isotopes. This assumption, together with the SM calculations, led us to assign spins.

\section{SUMMARY}

A wide range of $\mathrm{Cl}$ isotopes has been investigated by exploiting the mechanism of multiple nucleon transfers in the ${ }^{40} \mathrm{Ar}+{ }^{208} \mathrm{~Pb}$ reaction. This mechanism produces moderately neutron-rich nuclei with yields sufficient for studies via particle- $\gamma$ coincidences, a method which allows us to identify nuclei with good mass and charge resolution and uniquely attribute their characteristic $\gamma$ rays. By consistently incorporating the new measurement with the already available information, and by comparing the data with large-scale shellmodel calculations, we proposed updated level schemes for the chlorine nuclei from ${ }^{37} \mathrm{Cl}$ to ${ }^{43} \mathrm{Cl}$. In general, the agreement between shell-model calculations and experimentally deduced energy levels turns out to be very good.

Based on the characteristics of the transfer mechanism and by studying the nuclear structure in nearby isotopes, the increased mixing of different proton configurations was discussed along the isotopic chain. While for the stable ${ }^{37} \mathrm{Cl}$ $(N=20)$ isotope shell-model wave functions were dominated by one component only, for the more neutron-rich isotopes this dominance is attenuated. In addition, the promotion of the neutrons to the $p_{3 / 2}$ orbitals, namely across the $N=28$ shell, becomes gradually more important. By inferring the components of the wave function, it turns out that the behavior of the $s_{1 / 2}$ and $d_{3 / 2}$ proton orbitals, which could also couple to the quadrupole excitation of the corresponding $\mathrm{S}$ core, is very relevant in the understanding of the highest spin states here identified. In the middle of the shell $(N=24)$ these orbitals become nearly degenerate and the reduced energy splitting increases the mixing of different proton configurations.

It is difficult to disentangle these effects from energy systematics only, therefore further understanding of these $\mathrm{Cl}$ isotopes and development of the collectivity in this mass and charge region would require additional measurements of other relevant properties, such as the strength of electromagnetic transitions.

\section{ACKNOWLEDGMENTS}

The authors are grateful to the LNL accelerator staff for providing us with the good quality Ar beam and the target laboratory for the excellent target preparation. This work was partly supported by the EC FP6 EURONS Contract No. RII3-CT-2004-506065, and by the Croatian MSES Grant No. 0098-1191005-2890. A.G. and E.F. acknowledge the support of MICINN, Spain, and INFN, Italy through the AIC-D2011-0746 bilateral action. A.G.'s activity has been partially supported by the Generalitat Valenciana and MINECO, Spain, under Grants No. PROMETEO/2010/101 and No. FPA201129854.
[1] O. Sorlin and M.-G. Porquet, Prog. Part. Nucl. Phys. 61, 602 (2008).

[2] T. R. Werner et al., Phys. Lett. B 335, 259 (1994).

[3] J. Retamosa, E. Caurier, F. Nowacki, and A. Poves, Phys. Rev. C 55, 1266 (1997).

[4] P. D. Cottle and K. W. Kemper, Phys. Rev. C 58, 3761 (1998).

[5] D. Sohler et al., Phys. Rev. C 66, 054302 (2002).

[6] P. D. Cottle and K. W. Kemper, Phys. Rev. C 66, 061301(R) (2002).

[7] Z. Dombrádi et al., Nucl. Phys. A 727, 195 (2003).

[8] O. Sorlin et al., Eur. Phys. J. A 22, 173 (2004).

[9] X. Liang et al., Phys. Rev. C 74, 014311 (2006).

[10] A. Gade et al., Phys. Rev. C 74, 034322 (2006).

[11] B. Bastin et al., Phys. Rev. Lett. 99, 022503 (2007).

[12] A. Hodsdon et al., Phys. Rev. C 75, 034313 (2007).

[13] Z. M. Wang et al., Phys. Rev. C 81, 054305 (2010).

[14] Z. M. Wang et al., Phys. Rev. C 81, 064301 (2010).

[15] O. Sorlin et al., Phys. Rev. C 47, 2941 (1993).

[16] H. Scheit et al., Phys. Rev. Lett. 77, 3967 (1996).

[17] T. Glasmacher et al., Phys. Lett. B 395, 163 (1997).

[18] L. Gaudefroy, Phy. Rev. C 81, 064329 (2010).

[19] C. Force et al., Phys. Rev. Lett. 105, 102501 (2010).

[20] L. Cáceres et al., Phy. Rev. C 85, 024311 (2012).

[21] R. Chevrier et al., Phys. Rev. Lett. 108, 162501 (2012).

[22] T. Otsuka, T. Suzuki, R. Fujimoto, H. Grawe, and Y. Akaishi, Phys. Rev. Lett. 95, 232502 (2005).
[23] S. Aydin et al., Phys. Rev. C 86, 024320 (2012).

[24] R. Broda, J. Phys. G 32, R151 (2006).

[25] L. Corradi, G. Pollarolo, and S. Szilner, J. Phys. G 36, 113101 (2009).

[26] S. Lunardi et al., Phys. Rev. C 76, 034303 (2007).

[27] S. Bhattacharyya et al., Phys. Rev. Lett. 101, 032501 (2008).

[28] S. Szilner et al., Phys. Rev. C 84, 014325 (2011).

[29] F. Recchia et al., Phys. Rev. C 85, 064305 (2012).

[30] A. M. Stefanini et al., Nucl. Phys. A 701, 217c (2002).

[31] S. Szilner et al., Phys. Rev. C 76, 024604 (2007).

[32] G. Montagnoli et al., Nucl. Instrum. Methods Phys. Res., Sect. A 547, 455 (2005).

[33] S. Beghini et al., Nucl. Instrum. Methods Phys. Res., Sect. A 551, 364 (2005).

[34] L. Corradi, A. M. Stefanini, C. J. Lin, S. Beghini, G. Montagnoli, F. Scarlassara, G. Pollarolo, and A. Winther, Phys. Rev. C 59, 261 (1999).

[35] S. Szilner et al., Phys. Rev. C 71, 044610 (2005).

[36] C. H. Dasso, G. Pollarolo, and A. Winther, Phys. Rev. Lett. 73, 1907 (1994).

[37] A. Gadea et al., Eur. Phys. J. A 20, 193 (2004).

[38] National Nuclear Data Center, http://www.nndc.bnl.gov/ensdf

[39] F. Nowacki and A. Poves, Phys. Rev. C 79, 014310 (2009).

[40] E. Caurier, F. Nowacki, and A. Poves, Nucl. Phys. A 742, 14 (2004).

[41] M. Bouhelal, F. Haas, E. Caurier, F. Nowacki, and A. Bouldjedri, Nucl. Phys. A 864, 113 (2011). 
[42] E. Caurier, G. Martínez-Pinedo, F. Nowacki, A. Poves, and A. P. Zuker, Rev. Mod. Phys. 77, 427 (2005).

[43] B. Fornal et al., Phys. Rev. C 49, 2413 (1994).

[44] M. Ionescu-Bujor et al., Phys. Rev. C 80, 034314 (2009).

[45] D. O'Donnell, Ph.D. thesis, University of the West of Scotland, 2008 (unpublished).

[46] J. Ollier, Ph.D. thesis, University of Paisley, 2004 (unpublished).

[47] X. Liang, Ph.D. thesis, University of Paisley, 2001 (unpublished).

[48] E. K. Warburton and J. A. Becker, Phys. Rev. C 40, 2823 (1989).

[49] E. K. Warburton, J. W. Olness, and G. A. P. Engelbertink, Phys. Rev. C 7, 170 (1973)

[50] X. Liang et al., Phys. Rev. C 66, 037301 (2002).
[51] J. A. Cameron and B. Singh, Nucl. Data Sheets 94, 429 (2001).

[52] J. Ollier et al., Phys. Rev. C 67, 024302 (2003).

[53] D. O'Donnell et al., Phys. Rev. C 81, 024318 (2010).

[54] E. K. Warburton and J. A. Becker, Phys. Rev. C 39, 1535 (1989).

[55] R. L. Kozub et al., Phys. Rev. C 37, 1791 (1988).

[56] D. P. Balamuth, U. J. Hüttmeier, and J. W. Arrison, Phys. Rev. C 48, 2648 (1993).

[57] J. A. Winger, P. F. Mantica, and R. M. Ronningen, Phys. Rev. C 73, 044318 (2006).

[58] J. J. Valiente-Dobon et al., Phys. Rev. Lett. 102, 242502 (2009).

[59] D. Mengoni et al., Phys. Rev. C 82, 024308 (2010).

[60] D. Montanari et al., Phys. Rev. C 85, 044301 (2012). 